\title{
Cross-phase-modulation-instability band gap in a birefringence-engineered photonic-crystal fiber
}

\author{
B. Kibler, ${ }^{1,}{ }^{*}$ F. Amrani, ${ }^{1}$ P. Morin, ${ }^{1}$ and A. Kudlinski ${ }^{2}$ \\ ${ }^{1}$ Laboratoire Interdisciplinaire Carnot de Bourgogne (ICB), UMR 6303 CNRS-Université de Bourgogne Franche-Comté, \\ F-21078 Dijon, France \\ ${ }^{2}$ Université Lille, CNRS, UMR 8523-PhLAM-Physique des Lasers Atomes et Molécules, F-59000 Lille, France
}

(Received 12 November 2015; published 28 January 2016)

\begin{abstract}
We report the cancellation of the cross-phase-modulation-instability (XPMI) gain over a large spectral window (which we term the XPMI band gap) in a highly birefringent photonic-crystal fiber with zero group birefringence. The XPMI ceases to occur when single-frequency pumping of orthogonally polarized modes takes place in such a spectral band gap whose frequency bandwidth depends on the pump power itself. The suppression of XPMI sidebands is confirmed experimentally when Raman scattering remains negligible. At high powers the Raman Stokes wave, generated by the pump, implies novel dual-frequency pump configurations with large group-velocity mismatch, thus leading to another type of Raman-induced XPMI sidebands. The experimental results are in good agreement with analytical phase-matching calculations and numerical simulations.
\end{abstract}

DOI: 10.1103/PhysRevA.93.013857

\section{INTRODUCTION}

Polarization-dependent nonlinear effects are often considered as potential limitations to coherent optical communication systems, in particular related to the nonlinear phenomenon known as cross-phase modulation (XPM). The Kerr nonlinearity through XPM can couple two or more optical fields having different wavelengths, or distinct polarization states, making them interact efficiently under appropriate conditions. When such an interaction among multiple optical fields is fully controlled, one discovers that several practical applications can be investigated to manage light propagation. The XPM-induced coupling is known to significantly affect nonlinear phenomena resulting from a balance between dispersion and self-phase modulation. It gives rise to new regimes of instabilities and new effects in pump-probe configurations [1]. As an example, the XPM-induced coupling between two orthogonally polarized modes can destabilize a quasi-continuous wave (cw), thus leading to vector modulation instability (MI). This vector nonlinear process exhibits flexibility of phase-matching conditions compared to its well-known scalar counterpart [1]. Vector (XPM-induced) MI has been studied extensively in the context of birefringent fibers [2]. In addition to fiber nonlinearity and group-velocity dispersion, typical features of vector MI strongly depend both on the birefringence properties (i.e., weakly, randomly varying, or strongly birefringent) of the fiber under study and on the pump polarization [1,2]. This process can involve coherent or incoherent interactions between polarization components as a function of the linear phase mismatch (i.e., phase birefringence). In the following we focus on the case of highly birefringent fibers. Vector MI is then usually termed cross-phase-modulation instability (XPMI) and typically corresponds to the generation of orthogonally polarized sidebands from a pump linearly polarized at $45^{\circ}$ to the principal axes of the fiber.

Experimental observation of XPMI was first reported in the 1990 s by using standard highly birefringent (HiBi) fibers $[3,4]$. However, during the past decade, photonic-crystal

\footnotetext{
*Corresponding author: bertrand.kibler@u-bourgogne.fr
}

fibers (PCFs) [5] have allowed the exploration of a large number of interesting regimes in which XPMI can also occur thanks to their outstanding flexibility in the design of linear and nonlinear characteristics [5-8]. In particular, Ref. [9] gives a recent overview of the multiple scalar and vector MI sidebands that can be simultaneously observed in a highly birefringent PCF. Generally, the frequency bandwidth of the XPMI gain is relatively narrow compared to the scalar one. This feature points out its high sensitivity to fluctuations of fiber parameters, which has hampered its observation in nonperfectly uniform PCFs [10]. Nowadays, PCF properties can be precisely tailored and controlled during the drawing process, thus allowing researchers to benefit from XPMI sensitivity for applications as a nonlinear fiber-optic sensor [11]. Indeed, PCFs have become a suitable platform to study XPMI at any wavelength, since they also offer extremely high built-in birefringence by breaking the standard perfect sixfold symmetric core and cladding structure [5,12]. The induced form birefringence then exhibits strong wavelength dependence [13]. More generally, any significant variation of fiber characteristics over a short wavelength range modifies the phase-matching diagram of XPMI. A detailed analysis of vector MI has already been proposed by taking into account the frequency dependence of the group-velocity dispersion (GVD) [14]. Multiple sideband pairs can then be generated due to the effect of higher-order dispersion terms [9]. But recently, sign inversion and the cancellation of the group birefringence have also been reported at specific wavelengths $[15,16]$, thus having potential interest for the PCF-based devices that rely on polarization-dependent nonlinear effects.

In this work, we investigate the XPMI process in the context of HiBi PCFs with cancellation of the group birefringence at specific wavelengths around $1.5 \mu \mathrm{m}$. In particular, we report the existence of a XPMI spectral band gap whose frequency bandwidth depends on the input pump power. It is worth mentioning that the possible appearance of a nonlinear spectral gap in which XPMI vanishes was already demonstrated almost 20 years ago by means of a dual-frequency pumping configuration with orthogonal polarizations in a birefringent step-index fiber [17]. This complex pumping scheme allowed compensation of the group-velocity mismatch (related to the 
fiber birefringence) with chromatic dispersion by fine tuning of the frequency difference between the two pumps. As a result, the nonlinearity was responsible for the formation of a spectral gap in the XPMI phase-matching conditions. Here, we demonstrate that such a XPMI spectral band gap can be simply obtained in a birefringence-engineered PCF under the single-frequency pumping configuration. The suppression of XPMI sidebands is confirmed experimentally when Raman scattering remains negligible. At high powers the Raman Stokes wave, generated by the quasi-cw pump, induces a dual-frequency pump configuration, thus leading to additional Raman-induced XPMI sidebands. Our findings are clearly of interest in the applicability of an alternate class of controlled-birefringence optical waveguides for potential nonlinear-optics applications [16,18-20]. Moreover, they will also be advantageous when the suppression or the fine control of polarization-dependent nonlinearities is desirable [21].

This work is organized as follows. In Sec. II, we give an overview of the impact of zero group birefringence on the XPMI process by means of theoretical calculations of the phase-matching diagram and numerical simulations based on coupled nonlinear Schrödinger equations (NLSEs). In Sec. III, we describe the specific PCFs and experimental setup used here, and we present the experimental results that confirm our predictions obtained in Sec. II. In Sec. IV, we discuss the impact of the Raman effect on XPMI phase-matching conditions in our birefringence-engineered PCFs. In Sec. V, we conclude this work.

\section{THEORY AND NUMERICAL SIMULATIONS}

To analyze the impact of zero group birefringence on the XPMI process induced by single-frequency pumping, we designed a suitable birefringence engineered photonic-crystal fiber. Our PCF design includes the following requirements: high phase birefringence to favor XPMI and zero group birefringence close to potential high-power quasi-cw laser sources around $1.5 \mu \mathrm{m}$, but also single-mode operation over the wavelength range of interest and weak longitudinal fluctuations. By using some of the features reported in Ref. [15], the suitable microstructure was identified by simulating fiber properties with a commercial mode solver based on a finiteelement method. The PCF consists of a standard triangular lattice of air holes. The hole diameter and pitch are respectively $d=0.65 \mu \mathrm{m}$ and $\Lambda=1.12 \mu \mathrm{m}$, except for two large holes on either side of the core whose diameter is $0.75 \mu \mathrm{m}$ [see the cross section of the PCF design in the inset of Fig. 1(a)]. This core asymmetry then involves distinct propagation parameters for the fundamental linearly polarized modes as shown in Fig. 1(a). The corresponding phase and group birefringence are then given in Figs. 1(b) and 1(c), respectively. We recall the basic relations for the phase birefringence $B=n_{\mathrm{eff}, x}-$ $n_{\mathrm{eff}, y}$, and the group birefringence $G=n_{g, x}-n_{g, y}$, where $n_{\text {eff }, x}, n_{\text {eff, } y}$ and $n_{g, x}, n_{g, y}$ are respectively the effective indices and the group indices of both orthogonal polarization modes, respectively. $B$ and $G$ are linked through $G=B-\lambda d B / d \lambda$, where $\lambda$ is the input wavelength. Here, the $x$ axis joining the two larger holes is the fast one. The value of phase birefringence is here on the order of $10^{-3}$, which is typical of HiBi fibers [1]. We note that $B$ and $G$ have opposite signs
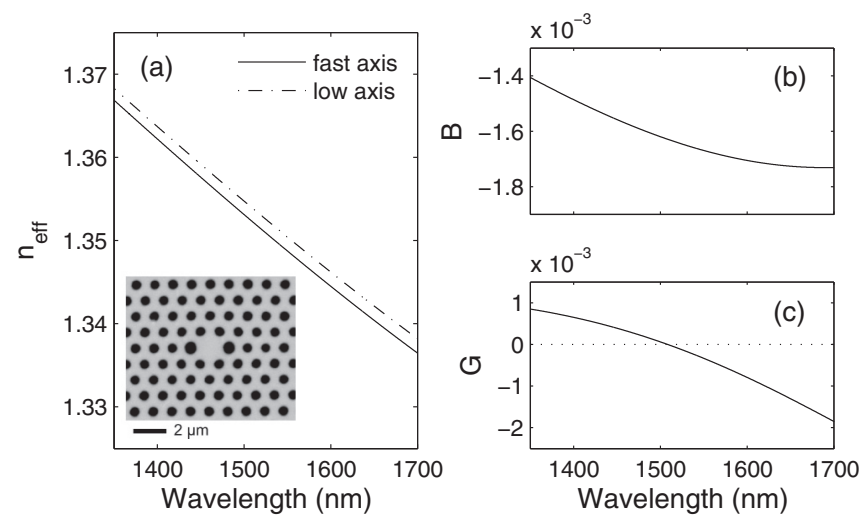

FIG. 1. Computed properties of the birefringence-engineered PCF: (a) Effective indices along the fiber principal axes. Inset: scanning electron microscope image of the typical PCF structure. (b) Phase birefringence. (c) Group birefringence. Note that the zero-group-birefringence wavelength is $1508 \mathrm{~nm}$.

at wavelengths below $1.5 \mu \mathrm{m}$ because $B / \lambda$ is smaller than $d B / d \lambda$. But, since the phase birefringence (in absolute value) increases with wavelength, one can reach a specific wavelength at which these two terms compensate, thus giving $G=0$. The numerical zero group birefringence is obtained at $1508 \mathrm{~nm}$ for this particular PCF design.

In a HiBi optical fiber, the interaction between two orthogonally polarized waves along $x$ and $y$ axes, and copropagating along the $z$ axis, is governed by the following two incoherently coupled NLSEs [1,14]:

$$
\begin{aligned}
& \frac{\partial A_{x}}{\partial z}+\frac{G}{2 c} \frac{\partial A_{x}}{\partial t}-\sum_{m \geqslant 2} i^{m+1} \frac{\beta_{m, x}}{m !} \frac{\partial^{m} A_{x}}{\partial t^{m}} \\
& =i \gamma_{x}\left(\left|A_{x}\right|^{2}+\frac{2}{3}\left|A_{y}\right|^{2}\right) A_{x}, \\
& \frac{\partial A_{y}}{\partial z}-\frac{G}{2 c} \frac{\partial A_{y}}{\partial t}-\sum_{m \geqslant 2} i^{m+1} \frac{\beta_{m, y}}{m !} \frac{\partial^{m} A_{y}}{\partial t^{m}} \\
& =i \gamma_{y}\left(\left|A_{y}\right|^{2}+\frac{2}{3}\left|A_{x}\right|^{2}\right) A_{y},
\end{aligned}
$$

where $A_{x}$ and $A_{y}$ are the slowly varying amplitudes of the linearly polarized field components along each axis, respectively. $\beta_{m x, y}$ and $\gamma_{x, y}$ are the dispersion coefficients and the effective Kerr nonlinear coefficients for both polarized waves. Note that $G / c=\beta_{1 x}-\beta_{1 y}$ is related to the group-velocity mismatch, where $c$ is the speed of light. The coefficients $\beta_{m \geqslant 2}$ are obtained from the expansion of $\beta_{2}(\omega)$ in a Taylor series around the pump frequency $\omega_{0}$, i.e., they describe the frequency dependence of the GVD. Figures 2(a) and 2(b) show the group-velocity dispersion and nonlinear parameter as functions of wavelength for the fundamental linearly polarized modes. For gain calculations, we consider averaged values of the dispersion terms and nonlinear parameter between the two polarized modes. Our PCF exhibits a large normal dispersion, which prevents any significant impact of higherorder dispersion terms in our analysis. Moreover, only vector sidebands will appear in this dispersion regime [1,9]. 
When considering a cw pump, the phase-matching diagram of both scalar and vector MI can be directly calculated through the linear stability analysis (LSA) of steady-state solutions of the NLSEs [Eqs. (1)]. Assuming that the pump (with a total power equal to $2 P_{0}$ ) is linearly polarized at $45^{\circ}$ with respect to the fiber axes, we can find the following stability matrix [14]:

$$
M=\left(\begin{array}{cccc}
\frac{-\Omega G}{2 c}+D_{+}+\gamma P_{0} & \gamma P_{0} & \frac{2}{3} \gamma P_{0} & \frac{2}{3} \gamma P_{0} \\
-\gamma P_{0} & \frac{-\Omega G}{2 c}-D_{-}-\gamma P_{0} & -\frac{2}{3} \gamma P_{0} & -\frac{2}{3} \gamma P_{0} \\
\frac{2}{3} \gamma P_{0} & \frac{2}{3} \gamma P_{0} & \frac{\Omega G}{2 c}+D_{+}+\gamma P_{0} & \gamma P_{0} \\
-\frac{2}{3} \gamma P_{0} & -\frac{2}{3} \gamma P_{0} & -\gamma P_{0} & \frac{\Omega G}{2 c}-D_{-}-\gamma P_{0}
\end{array}\right)
$$

Here we recall that $\Omega=\left(\omega-\omega_{0}\right)$, and we introduced the following parameter:

$$
D_{ \pm}=\sum_{m \geqslant 2}( \pm 1)^{m} \frac{\beta_{m}}{m !} \Omega^{m} .
$$

The four eigenvalues of the matrix $M$ are given by

$$
\begin{aligned}
K= & S_{-} \mp\left\{\Omega^{2} \delta^{2}+\frac{S_{+}^{2}}{4}+S_{+} \gamma P_{0} \mp\left[S_{+}^{2}\left(\Omega^{2} \delta^{2}+\frac{4}{9} \gamma^{2} P_{0}^{2}\right)\right.\right. \\
& \left.\left.+4 S_{+} \Omega^{2} \delta^{2} \gamma P_{0}\right]^{1 / 2}\right\}^{1 / 2},
\end{aligned}
$$

where $\delta=G / 2 c$, and $S_{ \pm}=D_{+} \pm D_{-}$. The gain spectrum of MI is simply obtained through the following relation $g(\Omega)=$ $2 \operatorname{Im}(K)$ [1]. To illustrate the existence of a XPMI spectral band gap in the following, we consider only the second-order dispersion term $\beta_{2}$, which gives $S_{-}=0$ and $S_{+}=\beta_{2} \Omega^{2}$.

Figure 3(a) reports the calculated MI phase-matching diagram with associated parametric gain as a function of the pump wavelength when using the fiber parameters described above (as an example $\beta_{2}=272 \mathrm{ps}^{2} / \mathrm{km}$ and $\gamma=33 \mathrm{~W}^{-1} \mathrm{~km}^{-1}$ at $1508 \mathrm{~nm}$ ) and a pump power $P_{0}=20 \mathrm{~W}$. The phase-matching diagram shows the suppression of XPMI sidebands for pump wavelengths around the zerogroup-birefringence wavelength. It also reveals the particular symmetric feature of the MI diagram with respect to this wavelength. The existence of such a XPMI spectral band gap originates from the fact that vector MI ceases to occur above a critical value of the input pump power [1]. This critical power $P_{0}=P_{c}=$ $3 G^{2} /\left(4 c^{2} \beta_{2} \gamma\right)$ is derived from the condition of MI gain in the normal-dispersion regime: $\operatorname{Im}(K)>0[1,3] . P_{c}$ depends on the group birefringence so that its value strongly decreases when $G$ becomes low enough (i.e., the group-velocity mismatch almost
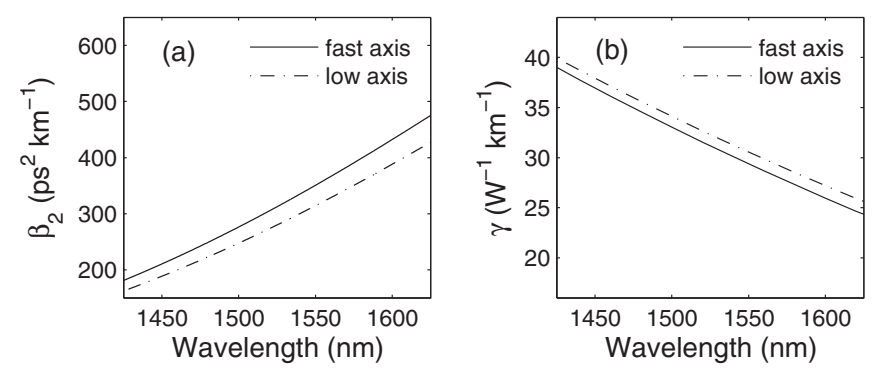

FIG. 2. (a) Group-velocity dispersion and (b) nonlinear parameter as functions of wavelength for the fundamental linearly polarized modes. vanishes [17]). As a result, the zero group birefringence implies here that the XPMI band gap does exist for any input power. For a fixed power, one can also determine the critical birefringence by using the relation given for $P_{c}$. Two distinct wavelengths corresponding to this critical birefringence can be found thanks to $G$-the curve plotted in Fig. 1(c). These critical wavelengths are reported with dashed lines in Fig. 3(a), thus revealing the frequency bandwidth of the XPMI band gap. From this simple analysis the spectral width of the band gap is expected to change when the input power is varied. Figure 3(b) confirms such a behavior, as we observe the increase of the band gap for higher input pump power $P_{0}=60 \mathrm{~W}$. The band-gap variation as a function of the input power $P_{0}$ (on each fiber axis) is depicted in Fig. 3(c).

To confirm the predictions provided by LSA about the existence of a nonlinear XPMI spectral band gap, we compare in Fig. 4 the above results with numerical simulations based on the coupled NLSEs [see Eq. (1)], taking into account the full dispersion curve. We considered a 12-m-long segment of the birefringence-engineered PCF pumped at two different wavelengths located inside or outside the predicted XPMI band gap. The $\mathrm{CW}$ pump is linearly polarized at $45^{\circ}$ with respect to the fiber axes with a total input power of $40 \mathrm{~W}$. As our analysis was performed at wavelengths for which the fiber exhibits a strong normal dispersion (i.e., without significant impact of higher-order dispersion), the agreement of XPMI gain features and orthogonally polarized sidebands between LSA and numerical simulations is excellent (see Fig. 4). Similar agreement was obtained when the input power, the pump wavelength, or the propagation distance was varied, provided that the development of XPMI gain bands remains restricted to the assumption of LSA. Note that in the normal-dispersion regime, the Stokes (anti-Stokes) sideband is polarized along the slow (fast) fiber axis.

\section{EXPERIMENTS}

To reveal the existence of the XPMI band gap, we used a typical configuration of vector MI experiments in optical fibers based on a quasi-cw pumping with a linear state of polarization oriented at $45^{\circ}$ with respect to the principal axes of the fiber under test, by using a polarizer and a half-wave plate. An analyzer was used at the fiber output to separately observe light polarized along the fast or the slow axis, and the output signal spectrum was recorded with an optical spectrum analyzer. The quasi-cw pump was a passively $Q$-switched laser that delivers $3.68 \mathrm{~ns}$ pulses at $1535 \mathrm{~nm}$ and $2.68 \mathrm{kHz}$ repetition rate. As the pump wavelength was fixed, several PCFs were 
(a)

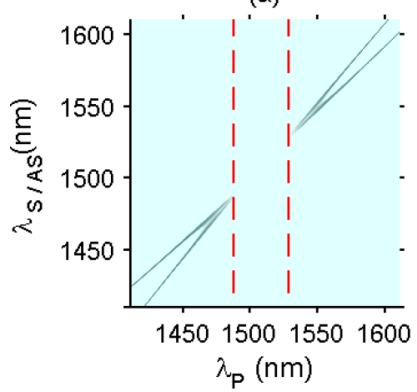

(b)

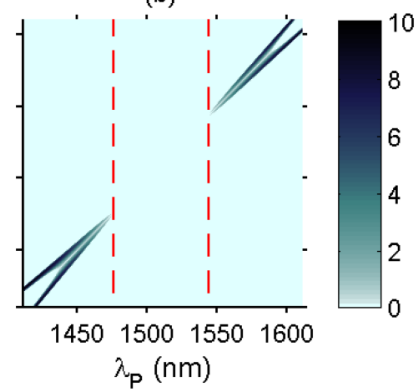

(c)

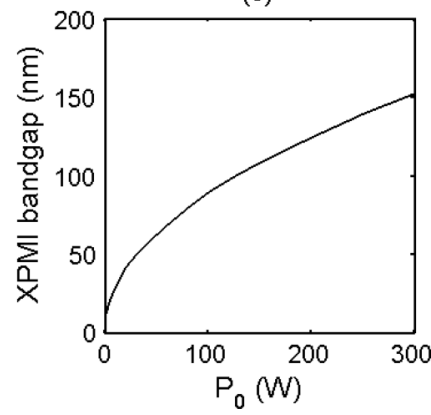

FIG. 3. (a),(b) Calculated MI phase-matching diagram with associated parametric gain $(\mathrm{dB} / \mathrm{m})$ as a function of the pump wavelength $\lambda_{P}$ for pump powers $P_{0}=20$ and $60 \mathrm{~W}$, respectively. The XPMI band gap for pump wavelengths is located between red dashed lines. (c) Corresponding evolution of XPMI band gap as a function of the input power $P_{0}$.

drawn with slight variations of the microstructure with regard to the PCF design described in the previous section. More precisely, the pitch $\Lambda$ was slightly varied (while keeping the $d / \Lambda$ parameter constant), which resulted in the shift of the zero-group-birefringence wavelength in the range 1450-1650 nm. Roughly speaking, a typical pitch variation of 5\% results in a variation of the zero-group-birefringence wavelength on the order of $100 \mathrm{~nm}$, which indicates that the latter it is extremely sensitive to the PCF design. In our experiment, we investigated three different PCFs (labeled A, B, and C) with a zero-group-birefringence wavelength located around the pump wavelength, at 1488,1540 , and $1573 \mathrm{~nm}$ respectively (see Fig. 5). The group birefringence and the second-order dispersion coefficient (not shown here) of fabricated PCFs were measured by standard techniques based on white-light interferometry. The dispersion at $1535 \mathrm{~nm}$ was found to remain highly normal for all the fibers, in the range 220-250 $\mathrm{ps}^{2} / \mathrm{km}$ (similar to our numerical design in Fig. 2). As a result, the wavelength position of XPMI band gaps (centered around the zero-group-birefringence wavelength) will differ for each PCF at a fixed input power, including or not the pump wavelength. As an example, the XPMI band gap is expected to extend over a few tens of nanometers for an input power of a few tens of watts [see Fig. 3(c)]. The length of fiber segments used for vector MI experiments was fixed to $12 \mathrm{~m}$.

The first set of XPMI experiments was performed by injecting a low peak power $P_{0}=38 \mathrm{~W}$ in the distinct fibers. For such a power, the LSA predicts the existence of the following wavelength ranges of the XPMI band gap: 1462-1510, 15171560, and 1554-1590 nm for PCFs A, B, and C, respectively. By consideration of our pumping wavelength located at $1535 \mathrm{~nm}$, XPMI is expected to be suppressed only in PCF B. The corresponding XPMI spectra recorded at fiber outputs are shown in Fig. 6. We compare the experimental results with numerical simulations based on the coupled NLSEs [see Eq. (1)] and with a cw pumping. An excellent agreement is obtained, thus confirming that XPMI is suppressed in fiber $\mathrm{B}$ thanks to the pumping in the vicinity of the zero group

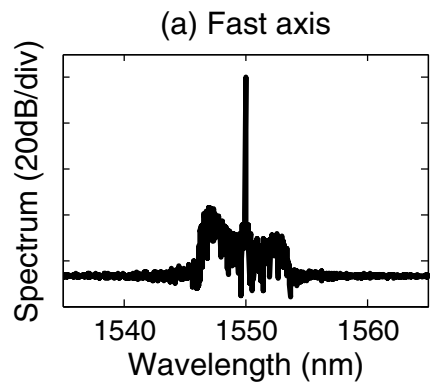

(d) Fast axis

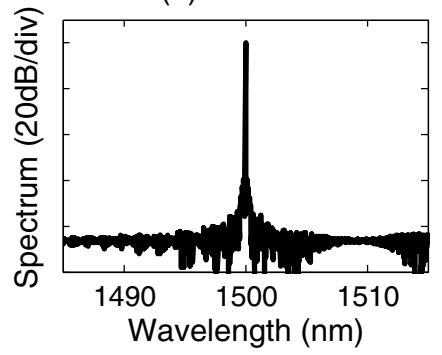

(b) Slow axis

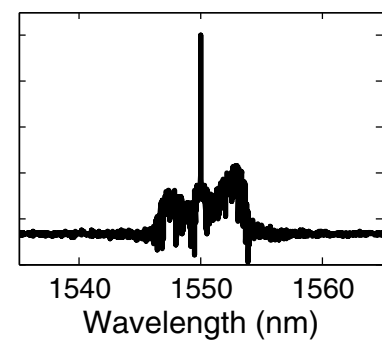

(e) Slow axis

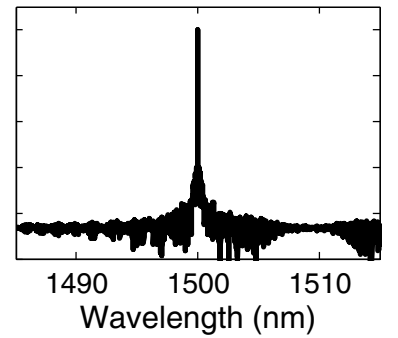

(c) Total intensity

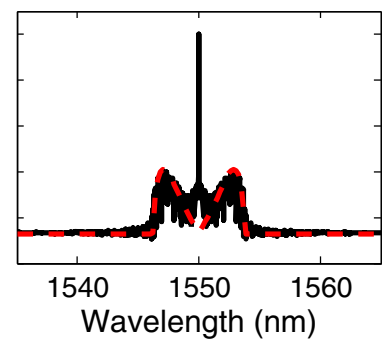

(f) Total intensity

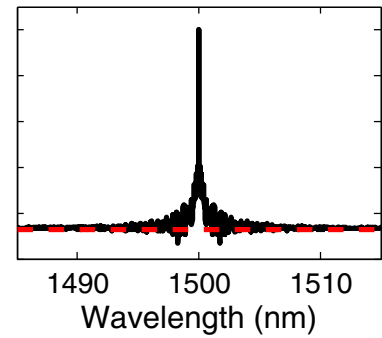

FIG. 4. Output spectra after $12 \mathrm{~m}$ of propagation in the birefringence-engineered PCF obtained by numerical simulations based on the coupled NLSEs [see Eq. (1)] and assuming a cw pump power of $40 \mathrm{~W}$ linearly polarized at $45^{\circ}$ with respect to the fiber axes. (a)-(c) Results obtained for a pump wavelength equal to $1550 \mathrm{~nm}$ outside the XPMI band gap. (d)-(f) Results obtained for a pump wavelength equal to $1500 \mathrm{~nm}$ inside the XPMI band gap. Red dashed lines correspond to the XMPI gain predicted by LSA [see Fig. 3(a)]. 


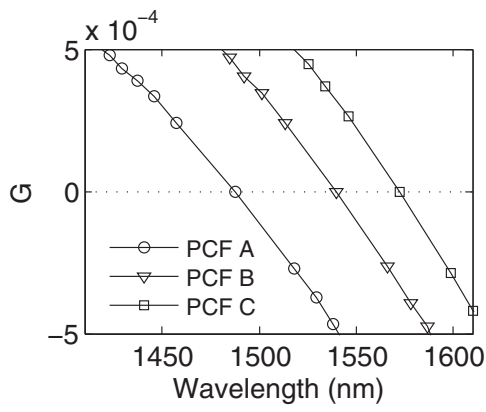

FIG. 5. Group birefringence measurements for the different PCFs used in the vector MI experiment.

birefringence (within the XPMI band gap). Note that measured fiber loss $\sim 30 \mathrm{~dB} / \mathrm{km}$ was introduced in the simulations. The vector nature of XPMI sidebands was carefully checked at the fiber output. As an example, Fig. 6(d) clearly shows the orthogonally polarized sidebands obtained along each axis of fiber $\mathrm{C}$, which is a typical feature of XPMI.

To extend the XPMI band gap over our pump wavelength in fibers $\mathrm{A}$ and $\mathrm{C}$, we have to increase the input peak power as discussed above in the theoretical section. With this aim, the suitable higher peak power has to be $P_{0}>170 \mathrm{~W}$, which could not be reached with our present setup. However, a second set of experiments was performed at intermediate powers, and the corresponding experimental output spectra are reported in Fig. 7. As expected from previous theoretical predictions, the XPMI band gap does not overlap with our pumping so that XPMI sidebands around the pump are still observed in fibers A and C. A detailed analysis of XPMI sidebands measured in fiber A confirms the slight decrease of their frequency shifts relative to the pump from 0.65 to $0.61 \mathrm{THz}$ when the input power is increased. This can be readily related to the extension of the XPMI band gap towards the pump wavelength which (a)

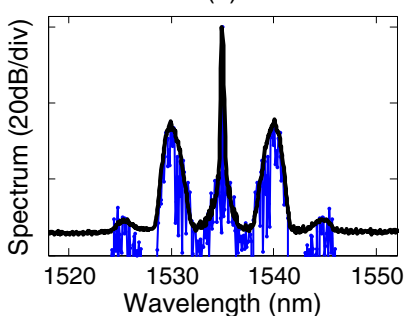

(c)

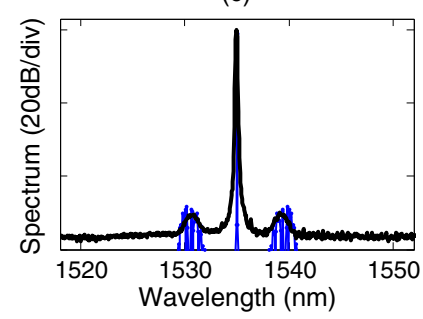

(b)

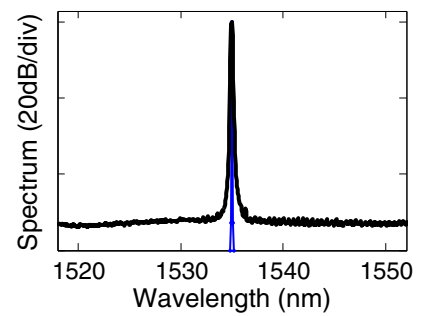

(d)

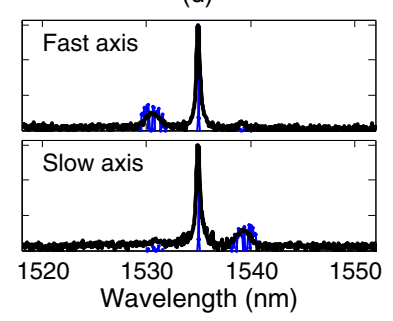

FIG. 6. Experimental XPMI spectra recorded at an input peak power $P_{0}=38 \mathrm{~W}$ for the different fiber segments (a) PCF A, (b) PCF $\mathrm{B}$, and (c),(d) PCF C. Blue curves with markers correspond to the numerical simulations based on the coupled NLSEs.
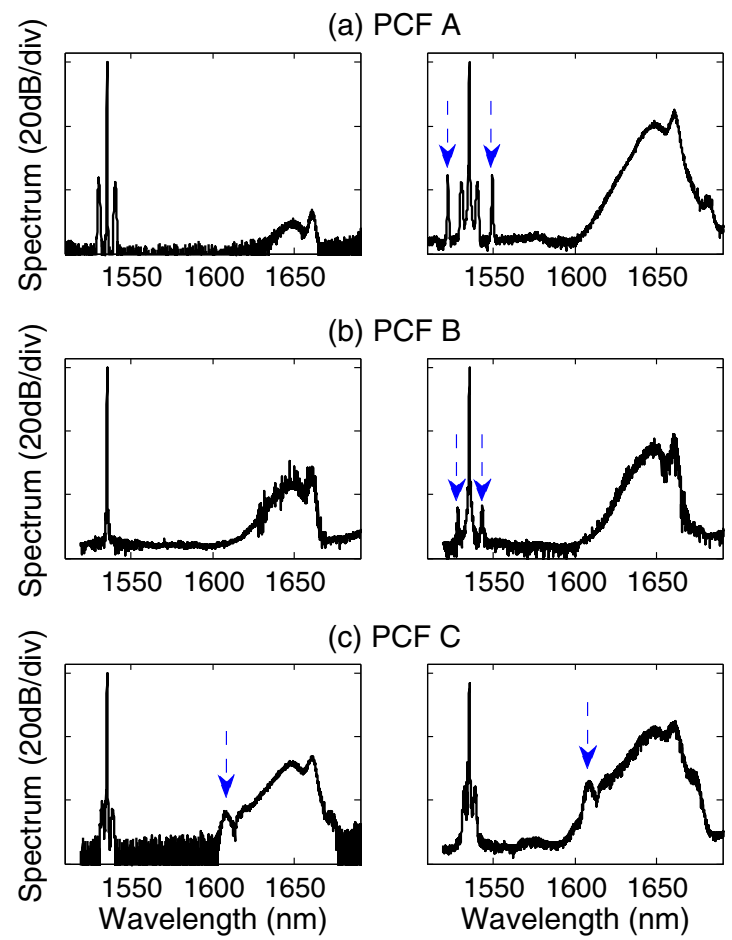

FIG. 7. Experimental spectra recorded for two input peak power ranges (left column) $P_{0} \sim 30-40 \mathrm{~W}$ and (right column) $P_{0} \sim$ 60-80 W at the output of (a) PCF A, (b) PCF B, and (c) PCF C. Note that a significant Raman Stokes pump induces the emergence of new Raman-induced XPMI sidebands (indicated by blue dashed arrows).

implies a lower frequency shift of XPMI sidebands (see Fig. 3). Moreover our measurements reveal the emergence of new sidebands strongly related to the presence of the strong Raman Stokes band generated around $1650 \mathrm{~nm}$ by the pump itself. When the Raman gain remains negligible or on the same order of magnitude as the XPMI gain (i.e., at low powers), these new sidebands do not appear, as shown in Fig. 7. For high powers, a new pumping configuration arises in our fiber with two pumps of different frequencies (i.e., the initial pump and the Raman Stokes pump) aligned with each of the two principal fiber axes (see the similar configuration in Ref. [22]), which allows novel phase-matching possibilities depending on the group-velocity mismatch between the two pumps (see the next section).

\section{DISCUSSION}

Our experimental results reveal that the Raman Stokes wave interacts with the pump to create a novel type of XPMI sideband with orthogonal polarizations as in Ref. [22]. Since both pump and Raman Stokes wave remain equally polarized at $45^{\circ}$ with respect to the fiber axes, they provide two distinct dual-frequency pump configurations. These are first (i) a pump component polarized along the fast axis associated with a Raman component polarized along the slow axis, and conversely (ii) a pump component along the slow axis and a Raman component along the fast axis. Each configuration is expected to generate its own pair of XPMI sidebands. In our case, the significant spectral width 
(a) Fast axis

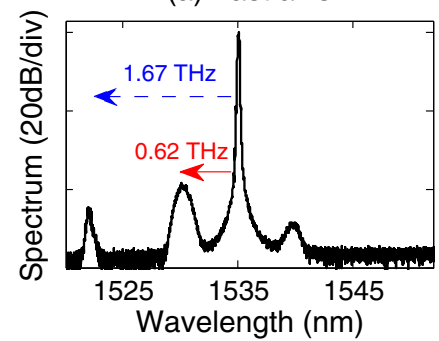

(b) Slow axis

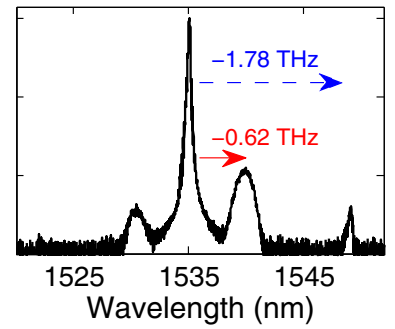

FIG. 8. Experimental output spectra with orthogonal polarizations (oriented along principal fiber axes) recorded for fiber A $\left(P_{0} \sim\right.$ $60 \mathrm{~W}$ ). XPMI sidebands induced by the initial $\mathrm{cw}$ pump (with balanced frequency shifts) are indicated by red solid arrows. The Raman-induced XPMI sidebands (with unbalanced frequency shifts relative to the pump) are indicated by blue dashed arrows.

of the Raman Stokes wave can mask some of the sidebands. Raman-induced XPMI sidebands are usually characterized by a narrow gain bandwidth and asymmetrical frequency shifts relative to the pump, in strong contrast to those obtained in the single-frequency cw pumping. As an example, Fig. 8 confirms the features of Raman-induced XPMI sidebands observed in fiber A. Note that such sidebands observed around the pump also exhibit orthogonal polarizations. The anti-Stokes peak is generated by the dual-pump configuration (ii), whereas the Stokes peak is obtained from the configuration (i), as previously described above.

In our present study, the distinct observations of Ramaninduced XPMI gain bands cannot be accurately confirmed by the standard LSA of the steady states of coupled NLSEs similar to Eq. (1) (as in Refs. [17,22]). The dual-pump configuration here implies two different frequencies (i.e., pump and Raman Stokes) in a fiber whose birefringence is strongly wavelengthdependent (note that a birefringent step-index fiber was studied in previous works [17,22]). As a consequence, the groupvelocity mismatch [i.e., related to $G$ in Eq. (1)] between the two pump waves has to be considered as a wavelength-dependent parameter in our analysis. We present below numerical simulations based on a coupled system of generalized NLSEs taking into account the full propagation constants and Raman scattering, in order to confirm again the XPMI spectral band gap for the single-frequency pumping configuration and the emergence of new Raman-induced XPMI sidebands. We used the fiber properties derived from the numerical PCF design provided in Sec. II, in particular the effective indices along principal fiber axes shown in Fig. 1. Figure 9 shows the numerical results obtained for two pump wavelengths 1535 and $1550 \mathrm{~nm}$ and a cw pump power $P_{0}=50 \mathrm{~W}$ (higher than in Fig. 4) to generate a significant Raman Stokes wave in both cases. We clearly observe the Raman-induced XPMI sidebands with narrow gain and unbalanced frequency shifts around the pump (about 0.05-0.06 THz difference between Stokes and anti-Stokes shifts). Besides, the sideband shifts also depend on the initial pumping wavelength (about $0.13 \mathrm{THz}$ difference between 1535- and 1550-nm pumping). All this confirms that the group-velocity mismatch between the input pump and the Raman Stokes pump plays a major role in the XPMI phase matching. These numerical results look very

(a) $\lambda_{\mathrm{P}}=1550 \mathrm{~nm}, \mathrm{P}_{0}=50 \mathrm{~W}$

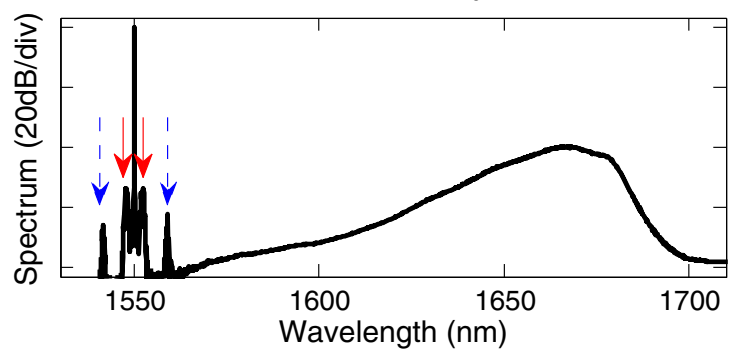

(b) $\lambda_{\mathrm{P}}=1535 \mathrm{~nm}, \mathrm{P}_{0}=50 \mathrm{~W}$

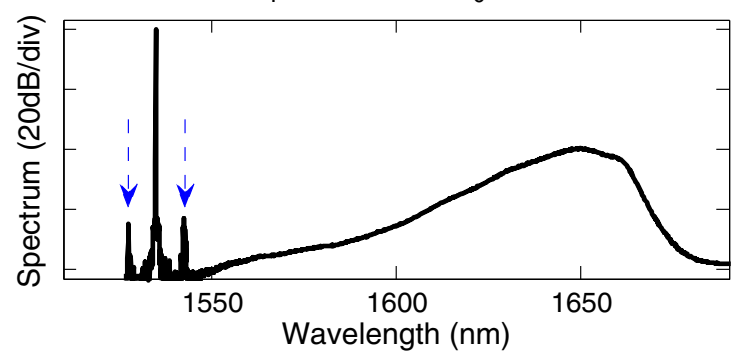

FIG. 9. Numerical spectra after $12 \mathrm{~m}$ of propagation in the birefringence engineered PCF designed in Sec. II and assuming a cw pump power of $100 \mathrm{~W}$ linearly polarized at $45^{\circ}$ with respect to the fiber axes (i.e., $P_{0}=50 \mathrm{~W}$ ). (a) Result obtained for a pump wavelength equal to $1550 \mathrm{~nm}$ outside the XPMI band gap. (b) Result obtained for a pump wavelength equal to $1535 \mathrm{~nm}$ inside the XPMI band gap. The XPMI sidebands (with balanced frequency shifts relative to the pump) are indicated by red solid arrows. The Raman-induced XPMI sidebands (with unbalanced frequency shifts relative to the pump) are indicated by blue dashed arrows.

similar to experimental spectra shown in Fig. 7 for fibers A and $\mathrm{B}$, since numerical parameters slightly differ from our experimental fiber (in particular the shifted position of the zero-group-birefringence wavelength). Note that when initial pumping occurs in the XPMI spectral band gap predicted from LSA in Sec. II (i.e., here only for $\lambda_{P}=1535 \mathrm{~nm}$ ) we confirm again that the balanced XPMI gain bands cease to occur [see Fig. 7(b)].

\section{CONCLUSION}

To conclude, we theoretically and experimentally demonstrated the existence of a XPMI spectral band gap in a birefringence-engineered photonic-crystal fiber, in particular when group birefringence cancels for orthogonally polarized modes. When single-frequency pumping takes place in such a spectral band gap, the XPMI gain is switched off. At high powers the Raman Stokes wave, generated by the singlefrequency pump itself, is shown to generate another type of XPMI sidebands in a dual-frequency pump configuration with large group-velocity matching. All these results may have important consequences for the design of PCF-based devices that benefit from or overcome polarization-dependent nonlinear effects. To predict the entire physical picture of complex XPMI phase matchings in such fibers requires the exact knowledge of wavelength-dependent propagation constants. 


\section{ACKNOWLEDGMENTS}

The authors thank G. Millot for stimulating discussions. B.K. and F.A. acknowledge financial support from the Conseil Régional de Bourgogne and the Labex ACTION
(Grant No. ANR-11-LABX-0001-01). A.K. acknowledges the "Fonds Européen de Développement Economique Régional," the Labex CEMPI (Grant No. ANR-11-LABX-0007), and Equipex FLUX (Grant No. ANR-11-EQPX-0017) through the "Programme Investissements d'Avenir."
[1] G. P. Agrawal, Nonlinear Fiber Optics, 5th ed. (Academic Press, Oxford, 2013).

[2] G. Millot and S. Wabnitz, Nonlinear polarization effects in optical fibers: Polarization attraction and modulation instability, J. Opt. Soc. Am. B 31, 2754 (2014).

[3] J. E. Rothenberg, Modulational instability for normal dispersion, Phys. Rev. A 42, 682 (1990).

[4] P. D. Drummond, T. A. B. Kennedy, J. M. Dudley, R. Leonhardt, and J. D. Harvey, Cross-phase modulational instability in highbirefringence fibers, Opt. Commun. 78, 137 (1990).

[5] P. St. J. Russell, Photonic-crystal fibers, J. Lightwave Technol. 24, 4729 (2006).

[6] D. Amans, E. Brainis, M. Haelterman, P. Emplit, and S. Massar, Vector modulation instability induced by vacuum fluctuations in highly birefringent fibers in the anomalous-dispersion regime, Opt. Lett. 30, 1051 (2005).

[7] J. S. Chen, G. K. Wong, S. G. Murdoch, R. J. Kruhlak, R. Leonhardt, J. D. Harvey, N. Y. Joly, and J. C. Knight, Crossphase modulation instability in photonic crystal fibers, Opt. Lett. 31, 873 (2006).

[8] S. Virally, N. Godbout, S. Lacroix, and L. Labonté, Two-fold symmetric geometries for tailored phasematching in birefringent solid-core air-silica microstructured fibers, Opt. Express 18, 10731 (2010).

[9] A. Kudlinski, A. Bendahmane, D. Labat, S. Virally, R. T. Murray, E. J. R. Kelleher, and A. Mussot, Simultaneous scalar and cross-phase modulation instabilities in highly birefringent photonic crystal fiber, Opt. Express 21, 8437 (2013).

[10] B. Kibler, C. Billet, J. M. Dudley, R. S. Windeler, and G. Millot, Effects of structural irregularities on modulational instability phase matching in photonic crystal fibers, Opt. Lett. 29, 1903 (2004).

[11] K. Tarnowski, A. Anuszkiewicz, J. Olszewski, P. Mergo, B. Kibler, and W. Urbanczyk, Nonlinear frequency conversion in a birefringent microstructured fiber tuned by externally applied hydrostatic pressure, Opt. Lett. 38, 5260 (2013).

[12] A. Ortigosa-Blanch, J. C. Knight, W. J. Wadsworth, J. Arriaga, B. J. Mangan, T. A. Birks, and P. St. J. Russell, Highly birefringent photonic crystal fibers, Opt. Lett. 25, 1325 (2000).
[13] G. Statkiewicz, T. Martynkien, and W. Urbańczyk, Measurements of modal birefringence and polarimetric sensitivity of the birefringent holey fiber to hydrostatic pressure and strain, Opt. Commun. 241, 339 (2004).

[14] F. Biancalana and D. V. Skryabin, Vector modulational instabilities in ultra-small core optical fibres, J. Opt. A: Pure Appl. Opt. 6, 301 (2004).

[15] P. Morin, B. Kibler, J. Fatome, C. Finot, and G. Millot, Group birefringence cancellation in highly birefringent photonic crystal fibre at telecommunication wavelengths, Electron. Lett. 46, 525 (2010).

[16] T. Martynkien, M. Szpulak, G. Statkiewicz-Barabach, J. Olszewski, A. Anuszkiewicz, W. Urbanczyk, K. Schuster, J. Kobelke, A. Schwuchow, J. Kirchhof, and H. Bartelt, Birefringence in microstructure fiber with elliptical $\mathrm{GeO}_{2}$ highly doped inclusion in the core, Opt. Lett. 33, 2764 (2008).

[17] P. Tchofo-Dinda, G. Millot, E. Seve, and M. Haelterman, Demonstration of a nonlinear gap in the modulational instability spectra of wave propagation in highly birefringent fibers, Opt. Lett. 21, 1640 (1996).

[18] A. Tonello, S. Wabnitz, T. Martynkien, G. Golojuch, and W Urbanczyk, Control of modulation and soliton polarization instabilities in photonic crystal fibers with birefringence management, Opt. Quantum Electron. 39, 435 (2007).

[19] K. Tarnowski, A. Anuszkiewicz, K. Poturaj, P Mergo, and W Urbanczyk, Birefringent optical fiber with dispersive orientation of polarization axes, Opt. Express 22, 25347 (2014).

[20] L. A. Fernandes, J. R. Grenier, P. V. S. Marques, J. S. Aitchison, and P. R. Herman, Strong birefringence tuning of optical waveguides with femtosecond laser irradiation of bulk fused silica and single mode fibers, J. Lightwave Technol. 31, 3563 (2013).

[21] A. Tonello and S. Wabnitz, Switching off polarization modulation instabilities in photonic crystal fibers, IEEE Photonics Technol. Lett. 18, 953 (2006).

[22] F. Amrani, B. Kibler, P. Grelu, S. Wabnitz, S. Trillo, and G. Millot, Cross-phase modulational instability induced by Raman scattering in highly birefringent fiber, Opt. Lett. 38, 5327 (2013). 